\title{
Programming Scalable Cloud Services with AEON*
}

\author{
Bo Sang \\ Purdue University \\ bsang@purdue.edu
}

\author{
Gustavo Petri \\ IRIF - Université Paris Diderot \\ gpetri@liafa.univ-paris- \\ diderot.fr
}

\author{
Masoud Saeida Ardekani ${ }^{\dagger}$ \\ Purdue University \\ msaeidaa@purdue.edu
}

\author{
Srivatsan Ravi \\ Purdue University \\ srivatsanravi@purdue.edu
}

\author{
Patrick Eugster \\ Purdue University, \\ TU Darmstadt \\ p@cs.purdue.edu
}

\begin{abstract}
Designing low-latency cloud-based applications that are adaptable to unpredictable workloads and efficiently utilize modern cloud computing platforms is hard. The actor model is a popular paradigm that can be used to develop distributed applications: actors encapsulate state and communicate with each other by sending events. Consistency is guaranteed if each event only accesses a single actor, thus eliminating potential data races and deadlocks. However it is nontrivial to provide consistency for concurrent events spanning across multiple actors.

This paper addresses this problem by introducing AEON: a framework that provides the following properties: (i) Programmability: programmers only need to reason about sequential semantics when reasoning about concurrency resulting from multi-actor events; (ii) Scalability: AEON runtime protocol guarantees serializable and starvation-free execution of multi-actor events, while maximizing parallel execution; (iii) Elasticity: AEON supports fine-grained elasticity enabling the programmer to transparently migrate individual actors without violating the consistency or entailing significant performance overheads.

Our empirical results show that it is possible to combine the best of all the above three worlds without compromising on the application performance.
\end{abstract}

\section{CCS Concepts}

-Computing methodologies $\rightarrow$ Distributed programming languages;

*Supported by NSF grants \# 1117065, \# 1421910, and \# 1618923, European Research Council grant \# FP7-617805 "LiVeSoft - Lightweight Verification of Software" and German Research Foundation under grant \# SFB-1053 "MAKI - Multi-mechanism Adaptation for Future Internet".

${ }^{\dagger}$ Now at Samsung Research America.

Permission to make digital or hard copies of all or part of this work for personal or classroom use is granted without fee provided that copies are not made or distributed for profit or commercial advantage and that copies bear this notice and the full citation on the first page. Copyrights for components of this work owned by others than ACM must be honored. Abstracting with credit is permitted. To copy otherwise, or republish, to post on servers or to redistribute to lists, requires prior specific permission and/or a fee. Request permissions from permissions@ acm.org.

Middleware'16, December 12-16, 2016, Trento, Italy

(c) 2016 ACM. ISBN 978-1-4503-4300-8/16/12 . \$ \$15.00

DOI: http://dx.doi.org/10.1145/2988336.2988352

\section{Keywords}

Cloud elasticity, actor system, strong consistency, scalability

\section{INTRODUCTION}

Providing cloud-based distributed solutions, and adequately leveraging the various capabilities provided by cloud providers is pivotal to many modern low-latency cloud-based applications and services. However, many of these applications (and services) still follow the de facto Internet architecture consisting of stateless front and middle tiers, equipped with a stateful storage tier at the back-end. Since most services must use this storage back-end, the scalability of the system as a whole is limited by the latency and throughput of the storage. To overcome this limitation, it is common practice to add a caching mechanism. While a caching middle tier might be effective in enhancing scalability, it comes at the cost of relaxing the concurrency control provided by the storage back-end. Moreover, this solution fails to exploit the inherent data locality of the application, since cache requests need to be shipped to other processes, potentially residing on a different virtual machine.

An alternative to the above architecture which has the potential to overcome these problems is to build a stateful middle tier using modern programming models based on actors. Actors encapsulate state and communicate with each other by sending events. In the actor model, consistency is guaranteed if each event only accesses a single actor, thus eliminating potential data races and deadlocks. Yet, this level of abstraction provided by many existing solutions (e.g., Erlang, Akka) is not appropriate for cloud-based programming since it is nontrivial to provide consistency for events spanning across multiple actors. Typically, the developer using these models still needs to deal with distributed systems and cloud programming issues such as asynchrony, failures and deadlock, to mention but a few.

Recent industrial and academic efforts have proposed actorbased frameworks (e.g., Orleans [8, 5] and EventWave [9]) for building and deploying cloud-based services. For example, Microsoft's Orleans is being used to implement many services, including Skype and the Halo game services [2].

All of these frameworks attempt to ensure a subset of the following properties: (i) Programmability: the simplicity of the framework is paramount to reduce the learning effort, increase developers' productivity, and guarantee the platform adoption. This aspect can be achieved by providing to the programmer the illusion of sequential semantics, hence 
ignoring the consistency challenges that may arise when the service runs in the cloud. (ii) Scalability: to effectively cope with unpredictable workloads, the framework - and in particular its runtime system - must be able to function at different scales; (iii) Elasticity: to achieve an economical solution, the framework must be able to automatically scale both in and out by adding and releasing resources to adapt to the workload at hand. Moreover, such workload adaptation should not violate application invariants or completely stall the computation.

This paper introduces AEON: a distributed framework that addresses the three concerns above as follows:

(i) To achieve programmability, AEON enables reasoning about multi-actor events with sequential semantics in mind. Specifically, AEON applications are modeled as a partiallyordered set of dynamically interacting contexts that, akin to actors, represent units of data encapsulation. Our protocol ensures that all the events are executed in an atomic and strongly consistent manner (à la strict-serializability in transactional systems). In other words, AEON provides to the programmer the illusion of a server answering to asynchronous requests one at a time in a sequential manner.

(ii) Partial-ordering of contexts in AEON induces an ownership network to organize contexts, whereby access to a context is only granted to the contexts that directly own it. This partial ordering results in a directed acyclic graph (DAG) of contexts that is the key for AEON to implement an efficient deadlock-free and starvation-free synchronization protocol. This protocol maximizes parallel execution of client request events, and is therefore highly scalable. This is in stark contrast to the synchronization employed in Orleans $[8,5]$, which does not provide strict serializability, or EventWave [9] which severely limits scalability by employing a global synchronization bottleneck.

(iii) As foundation for elasticity, AEONs runtime system allows for transparently migrating contexts across different servers of the system without affecting the semantics of the application, and thus dynamically adjusts the number of utilized virtual machines to the actual workload. Specifically, contexts can be automatically distributed across a data center without exposing the actual location of contexts in the network (i.e., it enforces location transparency [21]).

We have implemented a highly available and fault-tolerant prototype of $\mathrm{AEON}$ in $\mathrm{C}++$. Our empirical results show that it is possible to combine the best of all three worlds: programmability, scalability and elasticity without compromising on the application performance.

Concretely we make the following contributions: (1) After detailing challenges in developing elastic software in existing state-of-the-art paradigms such as EventWave [9] and Microsoft's Orleans [8], we present a novel programming model for building elastic cloud applications in a seamless and effortless fashion (§3). (2) The runtime of AEON implements a novel protocol for executing events in a strict serializable and highly scalable manner (§ 4) (3) AEON's runtime supports customizable automatic elasticity through the novel notion of an elasticity manager (§ 5). (4) We report an extensive evaluation, where we compare AEON against EventWave and Orleans on Amazon EC2 through a game application and the standard TPC-C benchmarks for transactional systems $(\S 6)$. Related work and final remarks are the subjects of $\S 7$ and $\S 8$ respectively.

The AEON code along with some extended details, in- cluding the operational semantics, are available on the AEON website: https://www.cs.purdue.edu/homes/bsang/aeon/

\section{OVERVIEW}

In this section, we first identify the challenges with programming support for scalable cloud services and applications and summarize the drawbacks of existing solutions to the problem. We then provide an overview of AEON, and illustrate how it addresses these challenges.

\subsection{Existing Work and Drawbacks}

There exist some efforts towards frameworks that help implement scalable elastic applications while reducing programming effort. EventWave [9] and Orleans [8] are two important works in this space.

Orleans. Orleans is an open-source framework, developed by Microsoft, based on the actor model. It introduces the concept of grains. Akin to actors, grains are single-threaded. There are two types of grains: stateful and stateless. Although Orleans was initially described to support transactions [8], the current open-source version does not provide transactional guarantees. However, for many cloud applications, transaction(al) execution is required for correctness since the manual implementation of distributed transactions always requires considerable effort. Moreover, it's easy to run into deadlocks in Orleans with (a cycle of) synchronous method calls because general grains are single-threaded and do not allow reentrance. Finally, re-distribution of grains is supported in Orleans, but the migration process provides no guarantees that the application semantics will be unaffected [27].

EventWave. EventWave is the nearest programming model to AEON in which applications are modeled as a tree of contexts. EventWave guarantees strict-serializability by totally ordering all requests at the (single) root context, assigning an unique id to each request and executing events in order of their ids. Consequently, EventWave provides only minimal progress [18]. This clearly limits scalability and overall performance, as adding more servers provides only limited benefits due to the bottleneck at the tree root. Moreover, EventWave only provides a simple API for the programmer to manually migrate contexts to specific servers by halting all executions during migration. This severely hampers elasticity and introduces a nontrivial performance degradation. EventWave also provides limited programmability since it organizes contexts strictly as a tree and does not support modification of tree edges. This prevents programmers from implementing classic distributed data structures such as Btrees and list-sets. $\S 7$ covers the drawbacks of other (perhaps less) related programming models for the cloud.

\subsection{AEON Overview}

Consider a massively multiplayer online (MMO) game, where players can circulate through an arena containing different buildings and rooms, each containing different objects. The players can interact with other players and objects in the same room. Such a MMO game has to process thousands of concurrent requests in an asynchronous environment, thus emphasizing the need for an efficient protocol to synchronize client requests. When there are too many online players and existing physical servers become contended, new servers must be allocated and some players must be mi- 
grated to those. Such players will still be interacting with other players and objects, and so the game service must handle the migrations both quickly and correctly.

Atomic Events and Ownership Network (AEON) is a general programming framework designed precisely to solve these problems. AEON allows the programmer to write applications assuming a sequential semantics. The AEON runtime system efficiently utilizes the distributed computing resources and supports seamless resource migration without sacrificing the application's correctness, thus relieving the application programmer of dealing with intricate concurrency issues.

Programmability. In Figure 1 we outline a simplified AEON implementation of our game. AEON takes an ObjectOriented (OO) approach to implement the server-side logic - the structure of the program follows a standard OO programming approach if we substitute the contextclass keyword by class, except for a few keywords that we will explain shortly in $\S 3$. Defining object structures as contextclasses instead of regular classes means that their instances will be automatically distributed, and relocated under workload pressure by the AEON runtime system as needed. Notice that the programmer does not need to implement any additional logic for the application to adapt to workloads.

For instance, suppose a client wants to put 50 gold coins into treasure from gold_mine. To this end, she issues a call of the form event player1.get_gold(50). The only difference between an event call and a normal remote method call is the event call decoration, which indicates to the runtime system that the call must be executed as an event. This annotation on the call site (as opposed to the method declaration) permits the reuse of methods, e.g., get_gold, both as events for client calls and as conventional synchronous methods in the case of another context calling it.

While asynchronous calls and events have been proposed before, the AEON programming model relieves the programmer from reasoning about race conditions, or tediously implementing synchronization mechanisms. AEON guarantees strict serializability. Therefore, events change the state of multiple contexts (i.e., instances of contextclasses) even residing on different machines, while maintaining the appearance of executing atomically and sequentially. In our example, an event call to updateTime0fDay in a Building context updates the time in all of the rooms before executing any subsequent event.

Scalability. In the interest of maximizing scalability, the programmer would like to execute requests from different users in parallel. However, it is not always the case that requests from different users operate on disjoint data. In the case where two or more requests operate on the same data, an efficient arbitration mechanism must be put in place to avoid strict serializability violations. Importantly, this mechanism should also avoid the possibility of deadlocks.

AEON employs a flavor of ownership types (akin to $[7$, 16] proposed for concurrent programming) to facilitate parallel yet atomic executions of distributed events: contexts form a directed acyclic graph (precisely, a join semi-lattice as detailed in § 3 ) structure indicative of their state sharing. Two events can run in parallel as long as they do not access shared portions of state. In AEON, a simple static analysis guarantees that the context graph derived from the context-accessibility (i.e. ownership hierarchy) between dif-

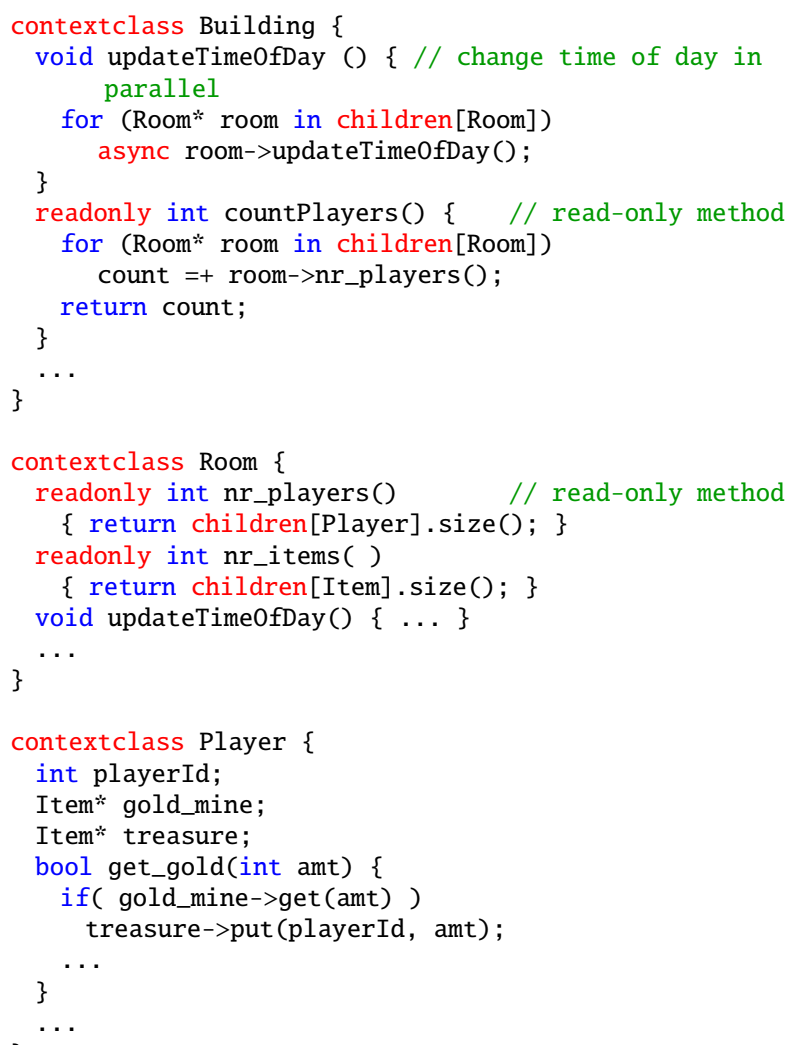

Listing 1: Simplified game example. Fields of contextclasses are not shown. Red keywords represent new AEON constructs.

ferent contexts is acyclic. In the example, we can see that a Player can own any number of Items, but not vice-versa.

Assuming two contexts of type Player sharing a common child of type Item, to guarantee the atomic execution of an event targeting one of the Player contexts, AEON delays the execution of events targeting the other Player until the former event is terminated. Otherwise, the shared Item context could be the source of data races, invalidating the serializable execution of both events. However, if two events are sent to Players in different Rooms, they can be executed in parallel without violating strict serializability of the system since they have no shared children. This enables a high degree of parallelization since a majority of events sent by different clients do not intersect.

Elasticity. To build a scalable distributed application that caters to dynamic workloads, the programmer would have to implement logic to: (i) migrate both data and computation between servers in case of a change in the workload; (ii) resolve which server has which pieces of data at any given time (which is non-trivial given that data might migrate); (iii) guarantee that ongoing requests are not disrupted by migrations. Writing even simple applications which meet the desired scalability criteria would require expert programmers in distributed systems, and even in that case it would remain an error-prone, time-consuming, and expensive endeavor. To avoid that such concerns related to distribution outweigh the concerns related to the actual program logic, AEON employs efficient migration protocols together with 


\begin{tabular}{c|c|c|c} 
& EventWave [9] & Orleans [8] & AEON \\
\hline Data encapsulation & Contexts & Grains & Contexts \\
Programmability restraint & Context tree & Unordered grains & Context DAG \\
Event consistency across actors & Strict serializability & No guarantees & Strict serializability \\
Event progress & Minimal(due to sequential bottleneck) & Possibility of deadlocks & Starvation-freedom [19] \\
Automatic elasticity & No & Yes [27] & Yes
\end{tabular}

Figure 1: Summary of distributed programming models for building cloud-based stateful applications

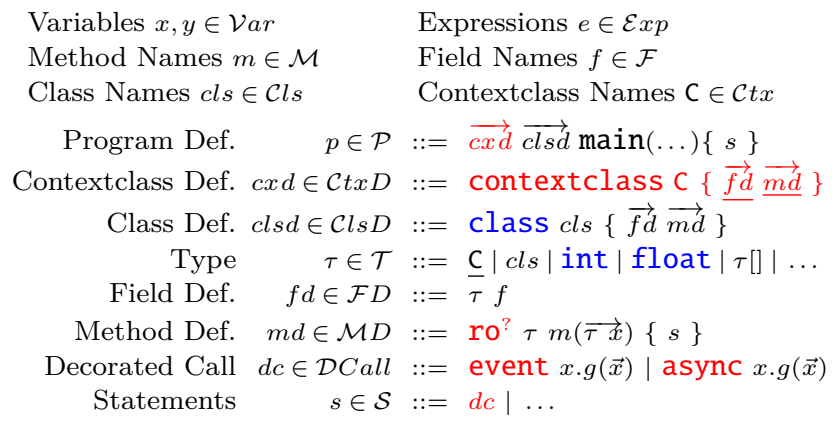

Figure 2: Syntax of AEON (excerpt). Underlined types are only allowed in the declarations of context fields and methods, not in class declarations.

an elasticity manager that enables the programmer to specify how contexts scale in/out. In our game example, the elasticity manager can easily move Room and Player contexts to different servers of the system when their current virtual machines become overloaded. For example, a player that starts a computation-intensive task might be migrated to a single virtual machine for the duration of the task. Figure 1 summarizes the properties provided by AEON with respect to Orleans and EventWave.

\section{PROGRAMMING MODEL}

In this section we describe the principal programming abstractions offered by AEON. Let us start by presenting a simplified abstract syntax of AEON in Figure 2. Notice first that AEON provides class declarations, as well as methods and fields like most mainstream OO programming languages. In addition, the language provides syntax for the declaration of contextclasses.

Classes and contextclasses. An AEON program comprises a series of contextclass declarations, a series of class declarations, and a main function which starts the execution of the AEON program. A context (an instance of a contextclass) is a stateful point of service that receives and processes requests either (i) in the form of events from clients, or (ii) in the form of remote method calls from other contexts. At a high level, a context can be considered as a container object or composite object that can be relocated between hosts. Contexts encapsulate local state (in the form of fields) and functionality (in the form of exported methods or events). In particular, AEON contexts hide internal data representations, which can only be read or affected through their methods.

Another aspect that distinguishes contextclass declarations from the standard class declarations is that types appearing in contextclass field and method declarations can also contain context-type expressions, underlined as $\underline{\tau}$ in
Figure 2. By inspecting the rule for types, we can see that contextclass names can thus be used as types only in contextclass level code, but not in normal classes. Thus, we vastly simplify the management of references (for example for garbage collection, in that passing an object by value does not implicitly create new references to contexts), and enable a simple static analysis to check that ownership respects a DAG structure as we shall describe shortly. Note that this restriction may be relaxed in future revisions of AEON.

Context ownership network. In a nutshell, AEON contexts are guarded by an ownership mechanism loosely inspired by the ones proposed in $[3,7]$. The concept of ownership allows AEON to establish a partial order among contexts (when considered transitively), and thus guarantees deadlock freedom when executing events.

We say that a context C is "directly-owned" by another context C' if any of the fields of C' contains a reference to C (we shall sometimes call the inverse relation of directlyowned "parent-child"). The ownership relation described above takes into account the transitive closure of the directlyowned relation. To the right of Figure 3, we depict a possible runtime ownership DAG for the application described in Figure 1. Here a Castle context of type Building owns two Room contexts: the Kings Room, and an Armory. In turn, each of the Rooms owns the respective Players currently in them, and a number of accessible Items. Players can also own Items. In addition, some contexts like Treasure can be owned by multiple contexts, Player1, Player2, and the Kings Room. Moreover, several contexts can own the same context, leading to a form of multi-ownership, which allows the sharing of state, a prevalent characteristic of object-oriented programming.

The ownership network enables the safe parallel execution of events provided that they do not access shared state. When multiple concurrent events can potentially access the same state, AEON serializes the events by exploiting the ownership network. The DAG structure of the ownership network guarantees that for any two contexts that might have a common descendant context, there exists an ancestor context that transitively owns both (we have a join-semilattice). ${ }^{1}$ In particular, for any set of contexts that have a common set of descendants, we are interested in the least common ancestor dominating them. Formally: for context $C$ in an ownership network G, assuming that $\operatorname{desc}(\mathrm{G}, \mathrm{C})$ represents the set of its descendant contexts, let share $(\mathrm{G}, \mathrm{C})$ be the set defined as follows:

$$
\begin{aligned}
& \text { share }(\mathrm{G}, \mathrm{C})=\left\{\begin{array}{l}
\left.\mathrm{C}^{\prime} \mid \operatorname{desc}(\mathrm{G}, \mathrm{C}) \cap \operatorname{children}\left(\mathrm{G}, \mathrm{C}^{\prime}\right) \neq \emptyset\right\} \cup \\
\mathrm{C}^{\prime} \mid \operatorname{desc}\left(\mathrm{G}, \mathrm{C}^{\prime}\right) \cap \operatorname{desc}(\mathrm{G}, \mathrm{C}) \neq \emptyset \&
\end{array}\right. \\
& \left.C^{\prime} \notin \operatorname{desc}(G, C) \& C \notin \operatorname{desc}\left(G, C^{\prime}\right)\right\}
\end{aligned}
$$

Then, we find in share $(G, C)$ all contexts which share a descendant context and are otherwise incomparable with C through

\footnotetext{
${ }^{1}$ Unnamed contexts are automatically added in the case of multiple maxima which share common descendants.
} 


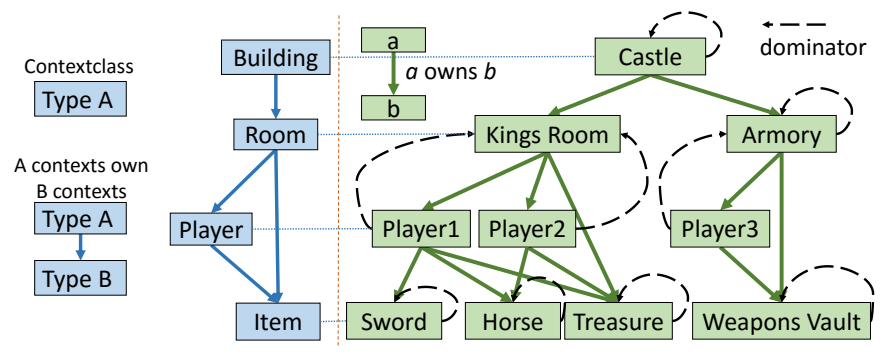

Figure 3: Game static and dynamic context structure.

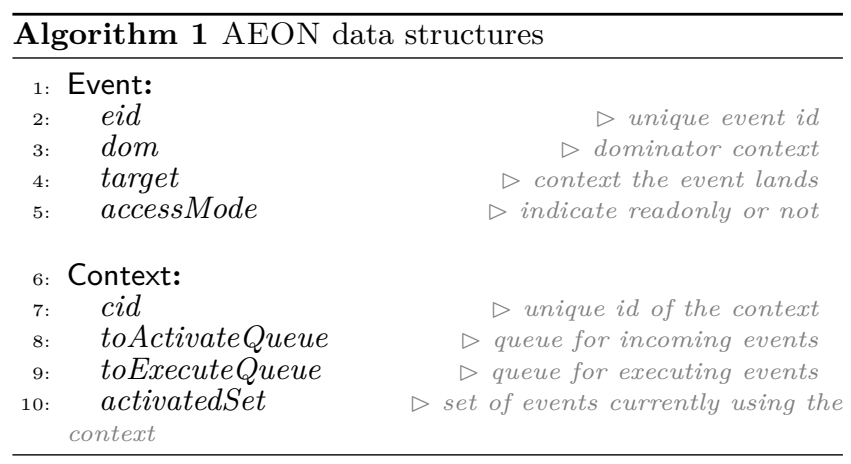

that directly export events, i.e., the entry points for clients to access the application).

In particular, since the directly-owned relation is related to referential reachability in the context-graph, we require that the graph of contextclasses reachable for a context that exports events be acyclic. An example of a hierarchy is shown in the left hand side of Figure 3, where the hierarchy represents essentially which contextclasses are contained in a certain contextclass.

To enforce this property, we put in place a simple analysis that collects for each contextclass method declaration, an over-approximation of the types of contexts that it could access. Since our language is in Administrative Normal Form [14], this can be done by a single pass over the declarations of contextclasses. Whenever a contextclass $\mathrm{c}_{0}$ declares an event that can use a contextclass $C_{1}$, we require that the contextclass $\mathrm{C}_{0}$ appears always at a higher level in the ownership network than $\mathrm{C}_{1}$ and we denote this constraint as $\mathrm{C}_{1} \leq \mathrm{C}_{0}$. The analysis succeeds if the collected constraints are acyclic except for the obvious reflexive cases (i.e., $c \leq c$ ), and rejects the program otherwise. This exception, made for reflexivity of the relation, allows for the construction of inductive data structures like linked-lists, or trees, at the slight expense of runtime checks upon modifications of context ownership structure. We note that the context ownership structure is modified when the object graph is explicitly modified.

\section{EXECUTION PROTOCOL}

In this section, we describe our novel synchronization protocol employed by AEON that arbitrates between two concurrent events to ensure strict serializability: the execution of an application's events built atop AEON appears like a sequential execution of the application that respects the temporal ordering of events. In other words, any AEON execution is indistinguishable from a valid sequential execution of the application' events. To synchronize among events that execute in contexts that have shared descendants, AEON employs the dominator context as a sequencer. Intuitively, when an event is launched in a context $C$ of an ownership network G, the dominator context of C (i.e. $\operatorname{Dom}(\mathrm{G}, \mathrm{C})$ ) is conceptually locked. An event locking a context has - conceptually - exclusive access to all the descendants of that context. Since we lock the dominator context, we have the guarantee that no other event that shares descendants with C starts its execution until the termination of the current event. These properties are ensured by AEON's implementation. 


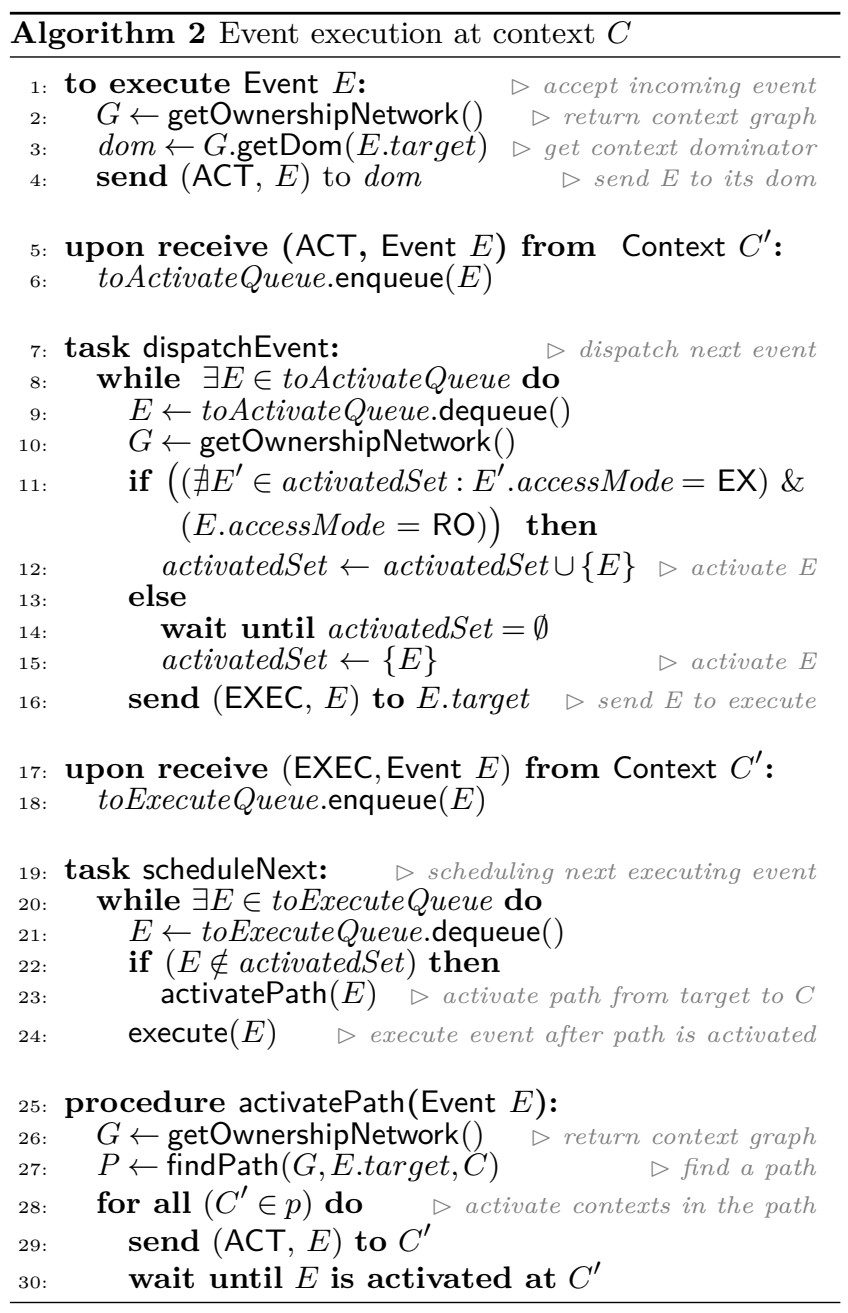

Protocol overview. Algorithm 2 provides high-level pseudocode of the AEON synchronization protocol and Algorithm 1 describes the data structures used in Algorithm 2. The execution of an event consists of method calls on the target context, or method calls on contexts that the target context owns. To execute, an event must take the lock on the target context. Each context has a set called activatedSet, which records events that currently lock that context. ${ }^{2}$ When an event tries to obtain the lock over the dominator of its target context, it will be placed into the dominator's toActivateQueue. When the event tries to lock a context other than the dominator, it must lock all events in a path from the dominator to itself in a top-down fashion. Finally, when a context method is called, the call is placed in the toExecuteQueue of the context based on the same ordering determined by the dominator.

Task dispatchEvent dequeues an event from toActivateQueue, and waits until the event obtains the lock, that is, it is added to the activatedSet. Note that multiple read-only events can hold the lock to a context at the same time. Once an event takes the lock, it is added to the toExecuteQueue for execution. Task scheduleNext is responsible for dequeuing an event from toExecuteQueue, and execute it.

When a method finishes its execution in a context, control

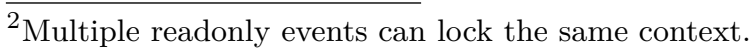

returns to the caller context, but it is not immediately removed from the context activatedSet; the removal happens only when the event has terminated in all contexts.

The execution model for method calls is by default synchronous, similar to Java RMI. However, in certain situations, for example when notifying every children of a certain context of a change, it is both unnecessary and inefficient to wait for the completion of a method call before issuing the following one, especially as these calls may be remote. The async method call decorator in AEON thus indicates that the execution of method call is asynchronous. This is the case of the calls to updateTime0fDay for the Room contexts in the method of the same name in the declaration of Building of Listing 1.

Evidently, in the case of multiple asynchronous methods that update the state of common children contexts, this behavior can lead to non-deterministism. This is analogous to data races which are considered a programming error, and have no semantics. In AEON this is also considered an error, albeit having a well-defined coarse-grained interleaving semantics (at the level of context accesses). In future work we will consider ruling out programs prone to this kind of error at compile-time.

We remark AEON employs a mechanism similar to readwrite locks exploiting the readonly annotations (cf. Figure 2). Unlike update events, which completely lock the target context, read-only events conceptually use a readlock (Line 11), so multiple read-only events can execute in parallel in the same context as detailed in Algorithm 2.

Informally, it is straightforward to see why the AEON protocol is strictly serializable. Specifically, let $\mathcal{A}$ be any application built using $\mathrm{AEON}$ and $\pi$ be any execution of $\mathcal{A}$. There exists a sequential execution $\pi^{\prime}$ of $\mathcal{A}$ equivalent to $\pi$ such that for any two events $E_{1}, E_{2}$ invoked in $\pi, E_{1} \rightarrow \pi E_{2}$ implies $E_{1} \rightarrow_{\pi^{\prime}} E_{2}$, where $E_{1} \rightarrow \pi E_{2}$ denotes the temporal ordering between events $E_{1}$ and $E_{2}$ in an execution $\pi$. Indeed, let $\mathrm{G}$ be any ownership network of an application $\mathcal{A}$ and $E_{1}, E_{2}$ be any two application events participating in an execution of the AEON protocol. Since G is a semilattice (cf. § 3 ), there is a deterministic monotonic ordering for $E_{1}$ (and resp. $E_{2}$ ) for conceptually locking the contexts accessed that begins with $\operatorname{dom}(\mathrm{G}, \mathrm{C})$ (and resp. $\operatorname{dom}\left(\mathrm{G}, \mathrm{C}^{\prime}\right)$, where $C$ (and resp. $C^{\prime}$ ) is the context on which $E_{1}$ (and resp. $\left.E_{2}\right)$ lands initially. Finally, locks on the contexts accessed during an event are released in the reverse order on which they are locked, thus ensuring starvation-freedom.

Illustration of event synchronization in AEON. We now illustrate the execution of the AEON synchronization protocol with our game example. Consider the single-owner case where a context $C$ is its own dominator, and an event is enqueued for execution at C. Castle and Armory in Figure 3 are examples of such single-owner contexts. In this scenario, to ensure that no two events modify context $\mathrm{C}$ or its descendants at the same time, events hold exclusive access of the context hierarchy starting at $\mathrm{C}$ during their execution, which is guaranteed by enqueuing all incoming events in the context's execution queue. Events execute once they reach the head of the queue.

However, if there is context sharing where the dominator of context C is a different context C', and the event is forwarded to C', it is sequenced at C', and starts its execution according to the sequence order. In Figure 3 all Player contexts are sharing contexts. The presence of sharing contexts 


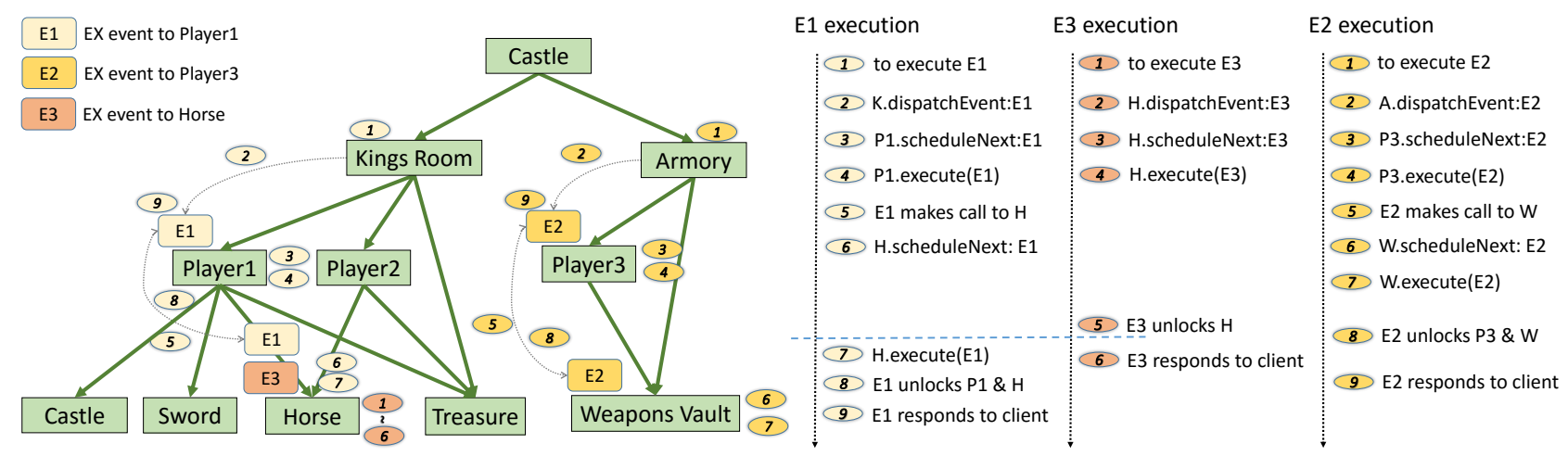

Figure 4: Event execution

introduces potential for deadlock. Consider for example the network in Figure 3, and assume that Player1 wants to steal the money from the shared Treasure, and then run away using the Horse. At the same time, Player2 wants to use the Horse to collect some debts, and then deposit the money in the Treasure. The schema delineated above can lead to a deadlock, where none of the players is able to execute their events. To avoid deadlocks, when an event with different target and dominator contexts is dispatched, AEON's runtime delivers that event to the dominator context: the event is serialized at the dominator context before being sent to its target context for execution. Therefore, in Figure 3, events targeting Player1 and Player2 need to be serialized in Kings Room, whereas events on Player3 are serialized in Armory (i.e., their dominator contexts). Observe that events targeting other contexts can safely execute in their target contexts.

We observe that for most cases, contexts have different dominators. For contexts that do not share sub-contexts with others, their dominators are themselves. Thus events to those contexts will be ordered independently. Generally, if two events are not ordered by the same dominator, they can execute in parallel.

Figure 4 shows a timeline of the execution of three events: $E_{1}$ targeting context Player1 (abbreviated P1 in the timeline); $E_{2}$ targeting Player3 (P3); and $E_{3}$ targeting Horse (H). The numbers in the timeline correspond to the numbered labels in the ownership graph in the left of the figure.

We can firstly observe that since the dominators of $E_{1}$ and $E_{2}$ are the Kings Room and the Armory respectively, and these contexts have no common descendants, they can execute completely independently and in parallel. The dominator context of event $E_{3}$ is the Horse, which is also its target. According to the rules outlined above, this event is immediately added to the toActivateQueue of Horse and subsequently activated. Importantly, event $E_{1}$ also requires to access Horse in the timeline. Therefore, when the execution of $E_{1}$ reaches the context Horse, the activatePath procedure will temporarily stall since in Horse $E_{3}$ is currently activated. Hence, $E_{1}$ has to wait for the completion of $E_{3}$ and the deactivation of $E_{3}$ in Horse before resuming its execution. In this way, the resulting serialization has the execution of $E_{3}$ before that of $E_{1}$, where the latter event sees the effect of the former one in the context Horse.

\section{ELASTICITY}

In this section, we explain AEON's elasticity manager called eManager. The eManager provides the following capabilities: (i) maintaining the global context mapping and ownership network, and (ii) managing context creation and migration based on elasticity policies. In our experiments, AEON is made fault tolerant using the Zookeeper service. In the remainder of this section, we explain the above two capabilities.

\subsection{Context Mapping}

Since contexts can dynamically migrate across hosts, and in order to deliver an event to the appropriate context, AEON first needs to find the host currently holding the corresponding context. To this end, every client and host caches the most recent context mapping that they have queried, and periodically refreshes their context mappings by querying the eManager. In practice, and in order to have a highly scalable and available system, clients and other hosts do not directly query the eManager. Instead, the eManager stores the latest context mappings along with the ownership network in a (configurable) cloud storage. Therefore, to locate a context for the first time (or in case the local cache has become invalid), a host or a client simply performs a read operation on the cloud storage system to retrieve the latest mapping. In the remainder of this paper, and for the sake of simplicity, we assume that clients and other hosts directly query the eManager.

\subsection{Elasticity Policy}

AEON gives the programmer the ability to define when and where contexts should be migrated. To this end, AEON employs an approach similar to Tuba [35, 34]. Every server periodically sends its resource utilization data (i.e., CPU, memory and IO) to the eManager. AEON provides a simple API to define when the eManager must perform a migration. The following example policies are implemented in AEON by default: (i) Resource utilization: in this policy, a programmer defines a lower and upper bound of a resource utilization along with an activation threshold. Thus, when a resource in a server reaches its upper bound plus a threshold the eManager triggers a migration. (ii) Server contention: under this policy, a programmer defines the total number of acceptable contexts per server. Hence, once a server reaches its maximum, the eManager triggers a migration. 
Once a migration is triggered, AEON computes a list of possible servers that can receive the contexts concerned. The default algorithm tries to move contexts from overloaded hosts to underloaded ones, but programmers can implement their own algorithms for choosing hosts and contexts. In addition, AEON allows programmers to define constraints on any attribute of the system similar to Tuba [35]. For instance, a constraint can disallow certain context migrations, or disallow a migration to a new host if total cost reaches some threshold.

Migration protocol. Once a migration is triggered, the eManager will follow the following atomic steps to migrate a context $\mathrm{C}$ from host $s_{1}$ to a new host $s_{2}$.

I The eManager sends a prepare message to $s_{2}$, notifying that requests for context $C$ might start arriving. Then, $s_{2}$ responds by creating a queue for context $C$ and acknowledges the eManager.

II Upon receiving the ack, the $e$ Manager informs $s_{1}$ to stop receiving events targeting $\mathrm{C}$ and it waits for $s_{1}$ ack.

III Once the eManager receives the ack, and after $\delta$ seconds, it updates its context mapping by assigning $C$ to $s_{2}$. Thus, from this point on, the eManager returns $s_{2}$ as the location of context $C$. It then sends a special event called migrate $\left(\mathrm{C}, s_{2}\right)$ to $s_{1}$ indicating that $\mathrm{C}$ has to be migrated to $s_{2}$.

IV Upon receiving migrate $\left(\mathrm{C}, s_{2}\right), s_{1}$ enqueues an event migrate $_{c}$ in C's execution queue. This event serves as a notification for context $\mathrm{C}$ that it must migrate. When migrate $_{c}$ reaches the head of C's queue, $s_{1}$ spawns a thread to move $\mathrm{C}$ to $s_{2}$.

$\mathrm{V}$ Upon completion of the migration, $s_{2}$ notifies the eManager that the migration is finished, and starts executing the enqueued events for context C.

Correctness under context migration. Observe that context C, at the end of step II when $s_{1}$ stops accepting events for $\mathrm{C}$ does not take any steps until step III when the $e$ Manager updates the context map. During this period, $s_{1}$ does not accept events targeting context $\mathrm{c}$, and eManager does not return $s_{2}$ as the new host for C.

Once the migration event enters $\mathrm{C}$ at $s_{1}$ for execution, it will be the only event that is being executed at C. Following the complete execution, both $s_{1}$ and $s_{2}$ will have up-to-date context mappings. If $s_{1}$ later receives an event for $\mathrm{C}$ from a host with stale context map, $s_{1}$ will forward those events to $s_{2}$ directly and notify source host to update its context map. In $\S 6$, we will evaluate the performance implication of halting the execution of events on a migrating context.

\subsection{Fault tolerance}

Similar to Orleans [8], AEON provides users with a special snapshot API that allows programmers to take consistent snapshots of a given context along with all its children. To this end, upon receiving a snapshot request for a context, the runtime of AEON dispatches a particular event called snapshot to that context. Consequently, this event takes consistent snapshots of that context and its children by getting contexts states, and writing them into a (configurable) cloud storage system like Amazon S3. To improve the performance, a programmer is able to override a method returning the state of a context. In case the overridden method returns null for a context, the runtime system will ignore that context during the checkpointing phase.
As we mentioned earlier, in practice the eManager is implemented as a stateless service that is responsible for updating context mapping and the DAG structure that are stored in a cloud storage system. The eManager also leverages the cloud storage system for persisting the steps of ongoing migrations. Therefore, if during the course of a migration, the eManager crashes, a newly elected eManager can read the state of an going migration, and tries to finish it. Details on how individual server and the eManager failures are treated without violating the consistency can be found on the AEON webpage.

\section{EVALUATION}

We implemented AEON on top of Mace [22], a C++ language extension that provides a unified framework for network communication and event handling. The implementation of AEON consists in roughly 10,000 lines of core code and 110 new classes on top of Mace. In the remainder of this section, we first compare scalability and performance of AEON with the two most closely related frameworks: EventWave [9] and Orleans [8]. We then study AEON's elasticity capabilities, and conclude the section by evaluating AEON's migration protocol and its effect on the overall throughput of the system.

\subsection{Scalability and performance}

In order to compare scalability and performance of AEON with EventWave and Orleans, we focus on the following two conventional metrics: (i) scaling out: how a system scales out as we increase the number of servers; and (ii) performance: how throughput changes with respect to latency as we increase the number of clients. To evaluate the above metrics, we implemented the TPC-C benchmark [1] and game application in all three systems.

To better study the effect of multiple ownership, the above two applications were implemented with and without multiple ownership. Throughout this section, we refer to the implementation with multiple ownership as AEON, and refer to the one without multiple ownership as $\mathrm{AEON}_{\text {so }}$ (for Single Ownership). Therefore, the programming effort for implementing the above applications is identical for $\mathrm{AEON}_{\text {so }}$ and EventWave.

We run AEON, $\mathrm{AEON}_{\text {so }}$ and EventWave on m3.large Linux VMs on EC2. For Orleans and Orleans*, we used m3.large Windows 2012 VMs on EC2.

\subsubsection{Game application}

Both EventWave and Orleans were previously evaluated using a game application similar to the example of $\S 2$. Therefore, we picked the very same game application described in EventWave [9]. Since EventWave does not support multiple ownership, the implementation does not allow Players to access Items directly. They could only access Items via Room. Since Orleans doesn't support transactional execution across multiple grains, we implemented two variants of game application in Orleans: (i) A version that ensures strict serializability. This version ensures Players access the shared Items atomically by means of locks. The Players simply lock the whole Room when they access their Items. This version is called Orleans in this section. (ii) Since one may argue that the above implementation is not the best possible algorithm for implementing the game in Orleans, we also implemented a non-strict serializability 


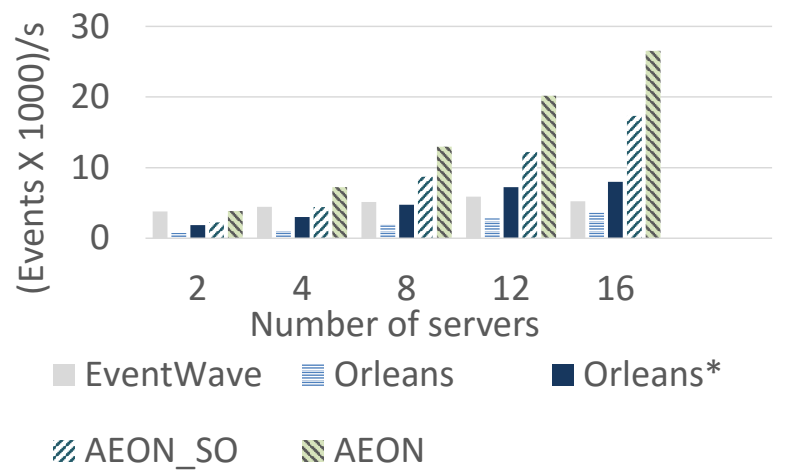

(a) Game Scaling out
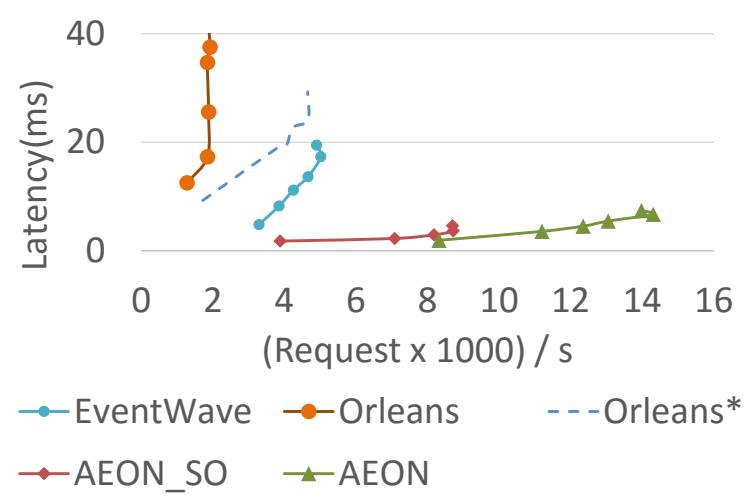

(b) Game Performance

Figure 5: Game application scalability and performance

variant of the game called Orleans ${ }^{*}$, in which Players just access their shared Items directly, and without synchronizing with other Players that have the same Items. This may result in incorrect executions potentially breaking application invariants. We note that this implementation is only used as a best-case scenario for the performance of Orleans, and it should otherwise be considered erroneous.

Scale out. Figure 5a shows scalability of different systems for the game application. In this experiment, we make each server hold one Room with fixed number of Items. So if there are more Players in one Room, Items will be shared by more Players.

As shown in Figure 5a, EventWave reaches maximum throughput with 12 servers since it needs to order all events in the root node. Observe that $\mathrm{AEON}_{\text {so }}$ (resp. AEON) outperforms EventWave by 3x (resp. 5x) when the number of servers reaches 16. Since both $\mathrm{AEON}_{\text {so }}$ and EventWave ensures strict serializability, and have identical tree structures, the $3 \mathrm{x}$ performance gain is not related to multiple ownership. Instead, the fact that in AEON events are not ordered at the root context along with async method calls lead to the observed substantial performance boost.

Interestingly, both $\mathrm{AEON}$ and $\mathrm{AEON}_{\text {so }}$ outperform Orleans* as well. This is because: 1) AEON is implemented in C++ and Orleans uses C\#. Hence, we expect AEON's implementation to have less overhead. 2) with the help of the ownership DAG, the runtime of AEON can optimize contexts placement, which will put Rooms, Players and Items in the Room on the same server. Orleans does not have similar rules, which may result in more message passing among servers. 3) due to the single-threaded nature of Orleans' grains, shared Items have to process requests from Players one by one. Though requests could be executed in parallel in Players, throughput is limited by the fixed number of Items within one Room.

Because of the parallelism provided by multiple ownership, we observe that AEON's performance is $50 \%$ more than $\mathrm{AEON}_{\mathrm{so}}$ when the number of servers reaches 16 . More precisely, since $\mathrm{AEON}_{\text {so }}$ does not have multiple ownership, in order to access Items belonging to a given Players, Room context needs to be locked. However, multiple ownership allows both Players and Room contexts to access Items thus leading to parallel execution of more events within one Room.
Performance evaluation. Figure $5 \mathrm{~b}$ plots throughput and latency of the game application when the number of servers is fixed to 8. Similar to Figure 5a, AEON outperforms all other systems. As we explained above, optimized AEON exploits allows for more parallelism and reduces the overhead in communication.

\subsubsection{TPC-C benchmark}

The TPC-C benchmark is an on-line transaction processing benchmark. TPC-C is a good candidate for comparing AEON with its rivals since it has multiple transaction types with different execution structures. Observe that transactions in TPC-C are similar to events in AEON and EventWave. All of our TPC-C implementations are made fault tolerant through checkpointing. We note that we used TPCC solely to stress-test AEON, and evaluate its performance under high contention. In reality, specifically engineered elastic distributed databases may be a better fit for serving TPC-C style applications.

The TPC-C benchmark implementation in AEON uses the following context declarations:

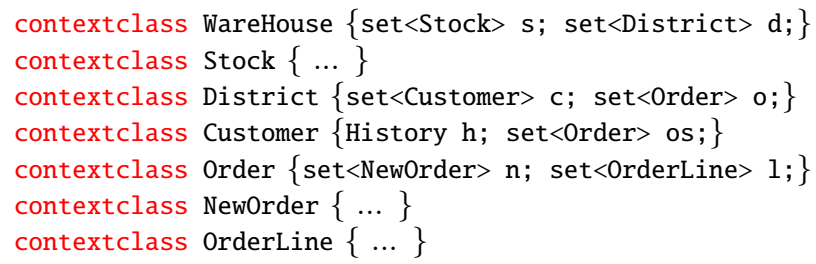

Since the number of items is fixed (i.e., 100K) in the TPC$\mathrm{C}$ benchmark, and does not need elasticity, warehouse and items form a single context.

Observe that an Order context has two owners: District and Customer. In our $\mathrm{AEON}_{\mathrm{so}}$ and EventWave implementations, and since they should follow a single ownership structure, the District context does not own the order context. In other words, the Order context is solely owned by the Customer context.

A typical approach for evaluating the scalability of a system using TPC-C is to partition TPC-C by warehouse, and put each warehouse on a single server $[10,37]$. But, as pointed out by $\mathrm{Mu}$ et al. [26], this approach does not stress the scalability and performance of distributed transactions (i.e., events in our programming model) because less than $\% 15$ 


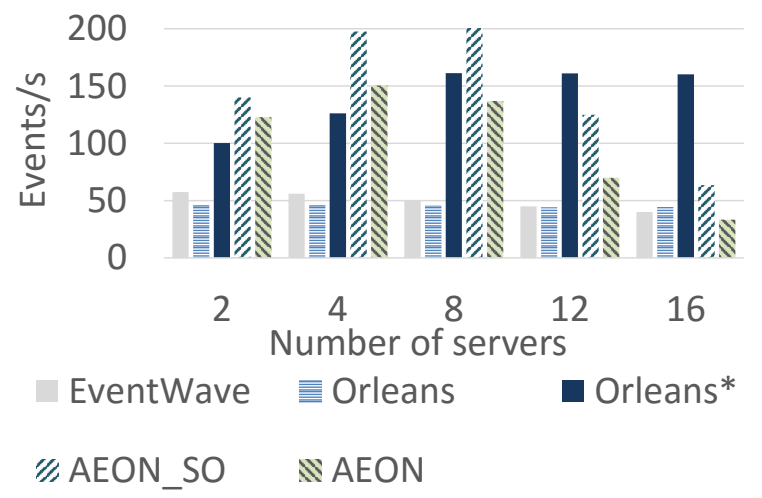

(a) TPC-C Scaling out

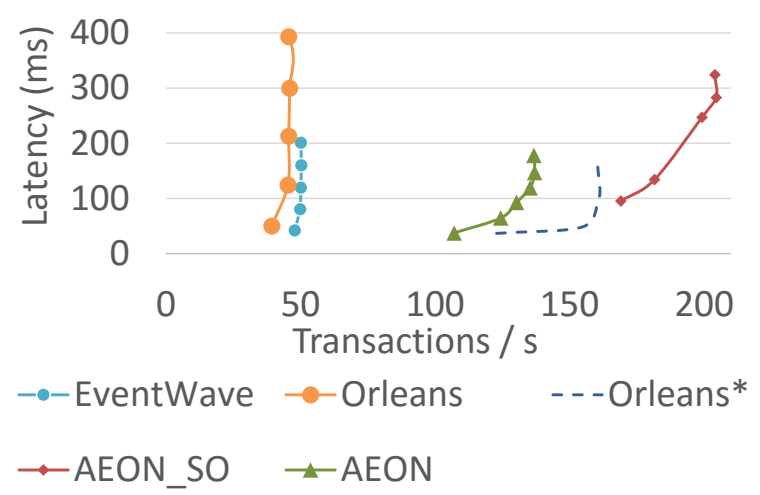

(b) TPC-C Performance

Figure 6: TPC-C scalability and performance

of transactions are distributed. Therefore, we also partition TPC-C by district similar to Rococo [26].

Similar to the game application, we also implemented two variants of TPC-C in Orleans: (i) A version that ensures strict serializability, which we shall name Orleans throughout this section. This version is implemented by exploiting the fact that stateful Orleans grains are single-threaded, and we orchestrate grains in a tree-like structure à la EventWave. (ii) We also implemented a non-strict serializability variant called Orleans*, in which the strict serializability is not guaranteed to be maintained. We note that this implementation is only used as a best-case scenario for the performance of Orleans, and it should otherwise be considered erroneous since it fails to ensure all the invariants of the TPC-C benchmark.

Scale out. Figure 6a plots scalability of different systems for the TPC-C benchmark. In this experiment, we placed one District (along with its corresponding Customers, Orders, etc.) in each server. While neither EventWave nor Orleans can scale as the number of servers increases, we observe that $\mathrm{AEON}$ scales up to 4 servers and $\mathrm{AEON}_{\text {so }}$ scales up to 8 servers. At this point, the Warehouse context becomes saturated, thus $\mathrm{AEON}$ and $\mathrm{AEON}_{\text {so }}$ cannot scale beyond 4 and 8 servers.

More specifically, $\mathrm{AEON}$ and $\mathrm{AEON}_{\text {so }}$ are able to outperform EventWave and Orleans due to (i) its use of the ownership network to order events, and (ii) async method calls inside events. As an event (i.e., a TPC-C transaction) finishes its execution in a parent context, it can continue its execution in a child context by using async method calls to the child context. For instance, once a payment transaction finishes its execution in a Warehouse context, it calls a method in a District context asynchronously, and releases the Warehouse context. This allows another event to enter the Warehouse for execution.

Figure 6a also shows that both Orleans* and $\mathrm{AEON}_{\text {so }}$ perform better than $\mathrm{AEON}$ when the number of servers reaches 16. This is because in TPC-C, multiple ownership does not help to increase the parallelism. Each District context owns several hundreds of Customer contexts and each Customer context owns several Order contexts. With multiple ownership, all these Order contexts are shared by both District context and Customer contexts. Consequently, method calls from
Customer contexts to Order contexts have to be synchronized by the District context, which is the dominator of Customer contexts. This leads to the District context becoming saturated fast. But, in the single ownership case, the dominators for Customer contexts are themselves. Therefore, the District context does not become the bottleneck.

Performance evaluation. Figure $6 \mathrm{~b}$ shows TPC-C performance boundaries with 8 servers. As expected, the throughput of EventWave and Orleans reach maximum with few clients (i.e., 4-8 clients) and then their latencies skyrocket immediately. This is due to both implementations failing to handle high contention at the Warehouse context properly. As shown in both Figure 6a and Figure 6b, Orleans* outperforms AEON with 8 servers since AEON has to pay extra overhead for strict serializability: events will be synchronized by District context.

\subsection{Elasticity}

As it was explained in $\S 5$, AEON has several built-in elasticity policies. In this section, we solely report our results on evaluating elasticity capabilities of AEON using the Service Level Agreement (SLA) metric as the elasticity policy of the game application.

For this experiment, we set the SLA for clients requests to $10 \mathrm{~ms}$. Therefore, AEON automatically scales out if it takes more than $10 \mathrm{~ms}$ to handle a client request. We placed our clients on 8 m1.large EC2 instances. Similar to Tuba [35], we varied the number of clients on each client machine from 1 to 16 according to the normal distribution. Therefore, at its peak time, there were 128 active clients in the system. The game application was deployed on a cluster of m1.small EC2 instances. To better understand the elasticity capabilities of AEON, we also run the game application with fixed numbers of servers (i.e., 8, 16 and 32 servers).

Figure $7 \mathrm{a}$ shows the average request latency that clients observed, and Figure $7 \mathrm{~b}$ plots the variation of the number of servers during the experiments. During peak times, both 8 -server and 16-server setups were unable to maintain the latency below $10 \mathrm{~ms}$. However, elastic and 32-server setups successfully met their SLAs. Due to migration cost, and fewer servers, clients in the elastic setup observed a slightly higher request latency.

Table 1 shows the percentage of client requests violating 


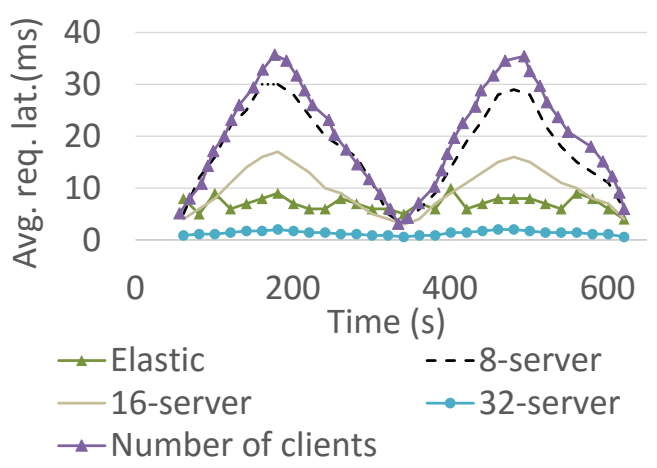

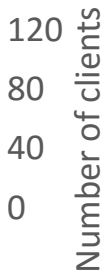

(a) Elastic v.s static

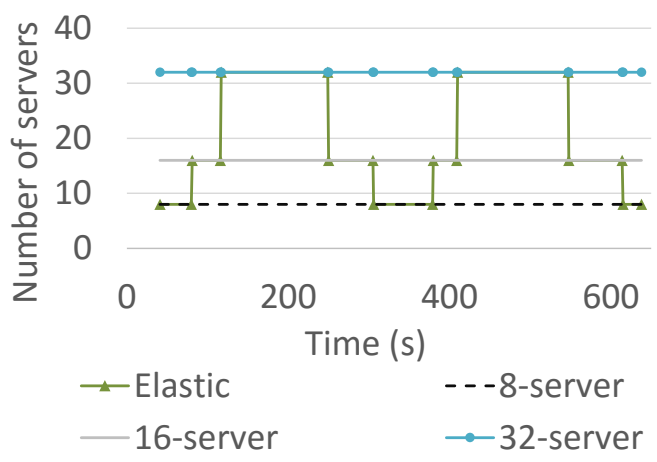

(b) Server number

Figure 7: Elastic game application

\begin{tabular}{c|c|c} 
Setup & $\%$ of requests $>10 \mathrm{~ms}$ & Avg. servers \\
\hline 8-server & $72.6 \%$ & 8 \\
\hline 16-server & $44.2 \%$ & 16 \\
\hline 22-server & $20.0 \%$ & 22 \\
\hline 32-server & $0.0 \%$ & 32 \\
\hline Elastic & $0.0 \%$ & 21.4
\end{tabular}

Table 1: Performance and cost

the SLA and the average number of servers used in each setup. While both 32 -server and elastic setups managed to meet the SLA, 32-server setup did so with $47 \%$ more resources. Lastly, observe that a (non-elastic) 22-server setup was unable to satisfy the SLA while the elastic setup fulfilled the SLA with 21.4 servers (on average).

\subsection{Migration}

In this section, we first study the effect of migration on the overall throughput of the system. We then evaluate the throughput of eManager when performing migration.

Overall throughput. We now show the effect of migration for different cases in our game application. In a first experiment, which we omit for lack of space, we migrated one context with different sizes up to $100 \mathrm{MB}$. Clearly, as a context's size increases, the time it takes to transfer from one server to another increases, but the overall throughput remains stable.

In the second experiment, we migrated different numbers

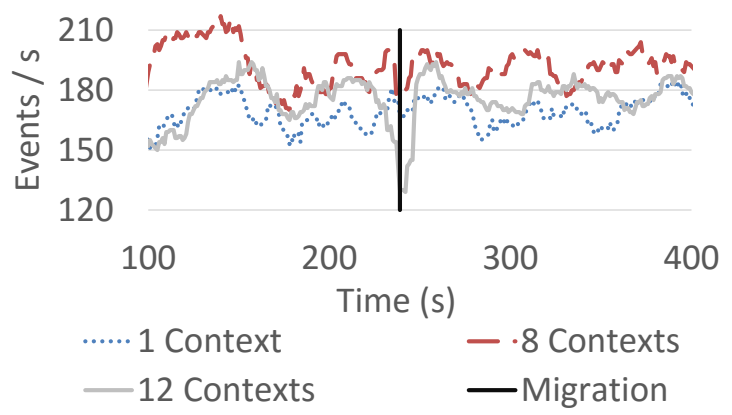

Figure 8: Migrating different number of contexts.

of contexts. Our experiments were deployed on EC2 with 20 m1.small instances. We create 20 Room contexts, one for each host. We also fixed the size of each context to $1 \mathrm{MB}$, and migrated contexts in order to determine the accumulated effect of multiple migrations at the same time - expected for high workloads.

Figure 8 shows the overall throughput variation of the system when migrating different numbers of Room contexts. The mild degradation observed, especially for the case of 12 simultaneous migrations is due to the fact that when a context is being migrated, requests to it are delayed for the duration of the migration. In this case more than 50 percent of the contexts are being moved, which should obviously impact the performance of the system for a short period of time as shown in the figure.

eManager throughput. Finally, we evaluated the maximum throughput of the migration algorithm introduced in $\S 5$ using a micro benchmark. To this end, the eManager moves contexts from one AWS instance to another. Figure 9 shows the eManager throughput with different context sizes. With m1.large instances, the eManager is able to move around 90 small contexts (i.e., $1 \mathrm{~KB}$ in size) or 40 large contexts (i.e., $1 \mathrm{MB}$ in size) every second. These numbers are dropped to $60 / 25$ with m1.medium instances, and $40 / 20$ with $\mathrm{m} 1$.small instances.

We expect that the number of contexts to be much less than the number of objects for an application. In other words, one context plays the role of a container for several objects as long as these objects do not require an independent elasticity policy. For example, consider the game application. Within a room, there can be several objects like lights and chairs. These objects can all be included in the Room context. However, in case light object has some non-trivial CPU or memory usage, it should be treated as a separate context.

\section{RELATED WORK}

Distributed programming models. The actor model $[29,8,5]$ is a popular paradigm that can be used to develop concurrent applications. Actors encapsulate state and execute code that can be distributed across multiple servers. Actors communicate with each other via message passing, and there is at most one thread executing in an actor at all times. This eliminates the complexities involved in guaranteeing data race and deadlock freedom. In that sense, actors are similar to contexts in our model. However, it is important to note that atomicity in actor systems is only given 


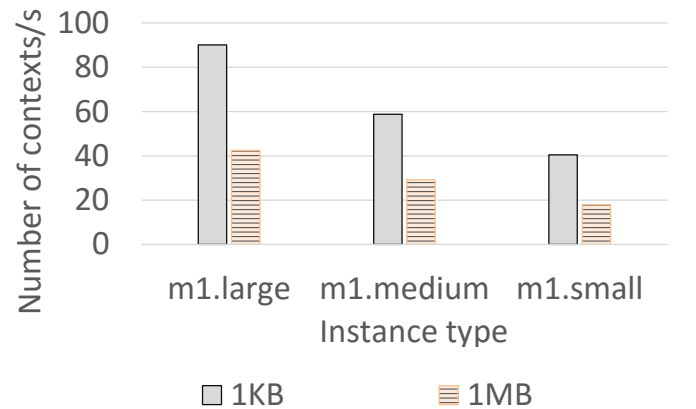

Figure 9: Max migration throughput on eManager

with respect to single actors, whilst an event in AEON can atomically modify several contexts.

Orleans [8] and EventWave [9], described in $\S 6$ provide concepts similar to AEON's contexts and events. The originality of AEON however resides in the ownership network, which allows us to guarantee strict serializability, unlike any of these two works, and deadlock freedom unlike Orleans, while still allowing sharing of state, and providing opportunities for automatic parallelization and scale adaptation. EventWave also induces single ownership and limits scalability by invariably synchronizing at a single root node.

Distributed transactional memory (DTM) [33] is a programming paradigm based on Transactional memory (TM) [36] that allows the programmer to build strictly serializable distributed applications with sequential semantics in mind, just as in AEON. However, to the best of our knowledge, there is no efficient DTM implementation that ensures strict serializability and provides implicit elasticity.

Transactors [13] have been proposed as a means to build distributed applications that provide strict serializability for events spanning multiple actors. However, transactors also do not provide support for building applications whose individual actors are distributed across the cloud. Moreover, there is no support for migrating actors without affecting consistency which is an important contribution from this work. We remark though that AEON may be thought of as an extension of transactors to the distributed cloud with support for automatic elasticity.

MapReduce [12] is a functional programming paradigm for the cloud that allows parallelizing computation via two sequential phases: map and reduce, to build applications involving huge data sets. However, writing a generic stateful application whose operations are non-commutative requires extensive synchronization among threads of computation, which is nontrivial to get right in the MapReduce paradigm [32], let alone supporting automatic elasticity.

AEON also shares similarities with models tailored to multi-core execution environments like Bamboo [39]. Bamboo provides a data-oriented approach to concurrency, where the programmer implements tasks, and the runtime system exploits dynamic and static information to parallelize data-independent tasks. Bamboo uses locks to implement a transactional mechanism for data-dependent tasks. Unlike AEON, Bamboo optimizes concurrency for multiple cores; distribution, migration, and scale adaptation are not considered.

In SCOOP [28], objects are considered individual units of computation and data. Separate calls - marked by the programmer - can be executed asynchronously from the main thread of execution. This is similar to the async calls of AEON. Similarly, separate calls can only be issued on arguments of a method, which is SCOOP 's way of controlling what AEON achieves through multiple ownership and events. SCOOP is not concerned with distribution or scale adaptation addressed by AEON.

Distributed programming languages. Emerald [20] is an $\mathrm{OO}$ distributed programming language, providing locality functionalities to allow programmers to relocate objects across the available servers. Unlike AEON, Emerald does not guarantee atomicity, and synchronization is left to the programmer. Moreover, Emerald was not designed for the cloud, where the existing resources might be unknown or dynamically allocated. Therefore, Emerald provides no elasticity. Identical arguments apply to programming languages like Erlang and Akka that contrast them from AEON and render them insufficient for building complex distributed applications with minimal programming effort.

Transactional key-value stores. Elastic databases (e.g., ElasTras [11], Megastore [4]) are similar to AEON: they partition and distribute data among a set of servers and provide consistency in the face of concurrent accesses. Unlike AEON, these do not provide a self-contained programming environment for writing generic elastic cloud applications.

Pilot job frameworks. A pilot job framework offers dynamic computational resources to a set of tasks $[6,15,24$, 31]. Applications running on such a framework can be split into a set of isolated tasks organized either as a "bag of tasks" $[6,15,24]$ or as a DAG workflow [31]. In the former case, tasks can execute in any order, while in the latter case, they should execute in a particular order defined by a DAG. These tasks are similar to the events of AEON, but unlike AEON where events can communicate with each other via contexts, tasks cannot communicate with each other.

Computation offloading. Offloading improves application performance by partitioning it among servers either at compilation or runtime $[17,23,25,30,38]$. Clearly, partitioning at compilation fails to provide elasticity. Dynamic partitioning, on the other hand, either targets singlethreaded applications, or requires an explicit addition of parallelism in contrast to AEON.

\section{CONCLUDING REMARKS}

We have presented the design and implementation of the AEON language. AEON provides a sequential programming environment for the cloud based on the standard paradigm of object-orientation. We provide a description of the semantics of AEON, and show that this semantics exploits parallelism while providing strict serializability as well as deadlock and starvation freedom. We have experimentally shown that the AEON runtime system scales as the number of client requests increases, and it is able to scale-out/in to provide an economic solution for the cloud. In future work we wish to lift some of the restrictions imposed on the usage of context references in classes and define a fine-grained elasticity policy language to allow the programmer control over the locality of contexts and usage of resources.

\section{Acknowledgments}

We thank the anonymous reviewers for their comments and suggestions that helped improving this work. 


\section{REFERENCES}

[1] TPC-C. http://www.tpc.org/tpcc/default.asp.

[2] Who is using Orleans. http: //dotnet.github.io/orleans/Who-Is-Using-Orleans.

[3] P. S. Almeida. Balloon types: Controlling sharing of state in data types. In Euro. Conf. on Object-Oriented Pging. (ECOOP), pages 32-59, 1997.

[4] J. Baker, C. Bond, J. C. Corbett, J. J. Furman, A. Khorlin, J. Larson, J.-m. L, Y. Li, A. Lloyd, and V. Yushprakh. Megastore: Providing scalable, highly available storage for interactive services. In Biennial Conf. on Innovative DataSystems Research (CIDR), pages 223-234, 2011.

[5] P. A. Bernstein, S. Bykov, A. Geller, G. Kliot, and J. Thelin. Orleans: Distributed Virtual Actors for Programmability and Scalability. Technical Report MSR-TR-2014-41, Microsoft, March 2014.

[6] B. Bode, D. M. Halstead, R. Kendall, Z. Lei, and D. Jackson. The Portable Batch Scheduler and the Maui Scheduler on Linux Clusters. In Annual Linux Showcase ES Conference (ALS), 2000.

[7] C. Boyapati, R. Lee, and M. C. Rinard. Ownership types for safe programming: preventing data races and deadlocks. In Conf. on Object-Oriented Prog. Sys., Lang. and Applications (OOPSLA), pages 211-230, 2002.

[8] S. Bykov, A. Geller, G. Kliot, J. R. Larus, R. Pandya, and J. Thelin. Orleans: cloud computing for everyone. In Symp. on Cloud Computing (SoCC), pages 16:1-16:14, 2011.

[9] W. Chuang, B. Sang, S. Yoo, R. Gu, M. Kulkarni, and C. E. Killian. EventWave: Programming Model and Runtime Support for Tightly-coupled Elastic Cloud Applications. In Symp. on Cloud Computing (SoCC), pages 21:1-21:16, 2013.

[10] J. Cowling and B. Liskov. Granola: Low-overhead distributed transaction coordination. In Usenix Annual Tech. Conf. (ATC), pages 223-235, June 2012.

[11] S. Das, D. Agrawal, and A. El Abbadi. Elastras: An elastic, scalable, and self managing transactional database for the cloud. Trans. on Database Sys., 38:5:1-5:45, 2013.

[12] J. Dean and S. Ghemawat. Mapreduce: Simplified data processing on large clusters. Commun. ACM, 51(1):107-113, Jan. 2008.

[13] J. Field and C. A. Varela. Transactors: A programming model for maintaining globally consistent distributed state in unreliable environments. In Symp. on Principles of Prog. Lang. (POPL), pages 195-208, 2005.

[14] C. Flanagan, A. Sabry, B. F. Duba, and M. Felleisen. The essence of compiling with continuations. In Conf. on Prog. Lang. Design and Implementation (PLDI), pages 237-247, 1993.

[15] J. Frey, T. Tannenbaum, M. Livny, I. T. Foster, and S. Tuecke. Condor-G: A Computation Management Agent for Multi-Institutional Grids. Cluster Computing, 5(3):237-246, 2002.

[16] D. Gordon and J. Noble. Dynamic ownership in a dynamic language. In Symp. on Dynamic Languages (DLS), pages 41-52, 2007.

[17] X. Gu, A. Messer, I. Greenberg, D. S. Milojicic, and
K. Nahrstedt. Adaptive Offloading for Pervasive Computing. IEEE Pervasive Computing, 3(3):66-73, 2004.

[18] R. Guerraoui and M. Kapalka. Principles of Transactional Memory,Synthesis Lectures on Distributed Computing Theory. Morgan and Claypool, 2010.

[19] M. Herlihy and N. Shavit. On the nature of progress. In Int. Conf. on Principles of Dist. Sys. (OPODIS), pages 313-328, 2011.

[20] E. Jul, H. M. Levy, N. C. Hutchinson, and A. P. Black. Fine-grained mobility in the emerald system (extended abstract). In Symp. on Op. Sys. Principles (SOSP), pages 105-106, 1987.

[21] R. K. Karmani, A. Shali, and G. Agha. Actor frameworks for the jvm platform. In Int. Conf. on Principles and Practice of Programming in Java (PPPJ), pages 11-20, Aug. 2009.

[22] C. E. Killian, J. W. Anderson, R. Braud, R. Jhala, and A. M. Vahdat. Mace: Language support for building distributed systems. In Conf. on Prog. Lang. Design and Implementation (PLDI), pages 179-188, 2007.

[23] Z. Li, C. Wang, and R. Xu. Computation offloading to save energy on handheld devices: a partition scheme. In Int. Conf. on Compilers, Architecture, and Synthesis for Embedded Sys. (CASE), pages 238-246, 2001.

[24] A. Luckow, L. Lacinski, and S. Jha. SAGA bigjob: An extensible and interoperable pilot-job abstraction for distributed applications and systems. In Int. Conf. on Cluster, Cloud and Grid Computing (CCGrid), pages 135-144, 2010.

[25] P. McGachey, A. L. Hosking, and J. E. B. Moss. Class Transformations for Transparent Distribution of Java Applications. Journal of Object Technology, 10:9: 1-35, 2011.

[26] S. Mu, Y. Cui, Y. Zhang, W. Lloyd, and J. Li. Extracting more concurrency from distributed transactions. In Symp. on Op. Sys. Design and Implementation (OSDI), pages 479-494, 2014.

[27] A. Newell, G. Kliot, I. Menache, A. Gopalan, S. Akiyama, and M. Silberstein. Optimizing distributed actor systems for dynamic interactive services. In Euro. Conf. on Comp. Sys. (EuroSys), pages 38:1-38:15, 2016.

[28] P. Nienaltowski, V. Arslan, and B. Meyer. Concurrent object-oriented programming on .NET. IEE Proceedings - Software, 150(5):308-314, 2003.

[29] M. D. Noakes, D. A. Wallach, and W. J. Dally. The J-Machine Multicomputer: An Architectural Evaluation. In Annual Int. Symp. on Computer Architecture (ISCA), pages 224-235, 1993.

[30] S. Ou, K. Yang, and A. Liotta. An Adaptive Multi-Constraint Partitioning Algorithm for Offloading in Pervasive Systems. In Int. Conf. on Pervasive computing and communications (PerComm), pages 116-125, 2006.

[31] I. Raicu, Y. Zhao, C. Dumitrescu, I. T. Foster, and M. Wilde. Falkon: a Fast and Light-weight tasK executiON framework. In Conf. on Supercomputing (SC), pages 43:1-43:12, 2007. 
[32] C. Ranger, R. Raghuraman, A. Penmetsa, G. Bradski, and C. Kozyrakis. Evaluating mapreduce for multi-core and multiprocessor systems. In Int. Symp. on High Performance Computer Architecture (HPCA), pages 13-24, 2007.

[33] P. Romano, L. Rodrigues, N. Carvalho, and J. Cachopo. Cloud-tm: Harnessing the cloud with distributed transactional memories. SIGOPS Oper. Syst. Rev., 44(2):1-6, Apr. 2010.

[34] M. Saeida Ardekani. Ensuring Consistency in Partially Replicated Data Stores. Ph.d., UPMC, Paris, France, Sept. 2014.

[35] M. Saeida Ardekani and D. B. Terry. A self-configurable geo-replicated cloud storage system. In Symp. on Op. Sys. Design and Implementation (OSDI), pages 367-381, Oct. 2014.

[36] N. Shavit and D. Touitou. Software transactional memory. In PODC, pages 204-213, 1995.

[37] A. Thomson, T. Diamond, P. Shao, and D. J. Abadi. Calvin : Fast distributed transactions for partitioned database systems. In Int. Conf. on the Mgt. of Data (SIGMOD), pages 1-12, 2012.

[38] L. Wang and M. Franz. Automatic Partitioning of Object-Oriented Programs for Resource-Constrained Mobile Devices with Multiple Distribution Objectives. In Int. Conf. on Parallel and Dist. Sys. (ICPADS), pages 369-376, 2008.

[39] J. Zhou and B. Demsky. Bamboo: a data-centric, object-oriented approach to many-core software. In Conf. on Prog. Lang. Design and Implementation (PLDI), pages 388-399, 2010. 